\title{
Digital Pheromone Scheme in Wireless Sensor and Robot Networks
}

\author{
L.Sravanthi ${ }^{1}$, D.V. Srihari Babu ${ }^{2}$ \\ ${ }^{1}$ L.Sravanthi, M.Tech (DECS), S.K.T.R.M.C.E., \\ ${ }^{2}$ D.V.Srihari Babu, M.Tech (Ph.D.), Assoc. Proff, S.K.T.R.M.C.E.,
}

\begin{abstract}
In this paper, we design a bio-inspired patrolling algorithm based on digital pheromone. As events have different importance, we refer to them as having different priorities. In our patrolling schemes, robots efficiently handle as many high priority events as possible. Inspired by the pheromone-based communication among ants, we introduce the concept of digital pheromones and propose a digital pheromone-based patrolling algorithm. Digital pheromones are introduced to denote the priority of an event. The main purpose of this algorithm is to lead robots to handle more events with higher priorities. Simulations demonstrate the impact of robots' patrol speed, speed of robots' handling events, etc.
\end{abstract}

\section{Introduction}

In our previous study, a divide-and-conquer based surveillance methodology is developed. A large region is divided into several small areas. Sensors, sinks, and robots are the main components of our surveillance system. Sensors, mainly for collecting data, report to their nearest sinks either periodically or only when something abnormal occurs. Different sensors are responsible for different tasks or targets. Sinks are responsible for collecting and managing regional information. Robots are responsible for handling events. They are patrolling all around inside the monitored area to collect most updated information from sensors and sinks. Robots can also help improve connectivity by collecting data from isolated sensors. Some sensors may be isolated or not connected to sinks. These sensors always buffer their data and wait for robots to collect them. Additionally, robots are equipped with sensors and RFIDs which can be deployed when necessary. Sensors can improve connectivity, and RFIDs can carry specific information. In the following contexts, we will go into further detail about these aspects. When several events are detected, a robot will figure out the most efficient path in order to handle the largest number of important events. A patrol algorithm is determinant for robots' working efficiency. In this paper, we propose a bio-inspired digital pheromone based patrolling algorithm. The algorithm can also be extended to several robots, considering the collaboration among robots. For example, two robots can share their information and collaborate to handle events efficiently. In, a dynamic itinerary planning for mobile agents in wireless sensor network is proposed. Although it is designed for an agriculture application with a special concern of crop monitoring and frost prediction, itinerary planning is a quite similar topic with our patrolling problem. Bio-inspired research has become a hot topic in computer science, especially in wireless networks. For example, scent-marking, an important communication way among many different mammals, especially primates, is deeply studied and we proposed a primate-inspired method for pursuing and tracking problem of robots in. Similar with scent-marking activity of primates, pheromones play a critical role in ants' communications. Ant Colony Optimization is a very interesting topic, which has been used as models for solving some combinational optimization problems. It is inspired by the social behavior of ants leaving pheromone trails for the colony to follow. An example of its application is to solve the traveling salesman problem in software, which allows the software to achieve the shortest route. Also inspired by ants using pheromone trails, an artificial ant colony approach to distributed sensor wakeup control (SWC) in wireless sensor networks (WSN) is presented in with the application for the joint task of surveillance and target tracking. In a routing algorithm in wireless Mobile Adhoc Networks (MANET) based on ants' activity is studied. Another example of bio-inspired application in wireless networks is an improved version of a swarm-intelligence ad hoc routing algorithm EARA. Other related papers include. Inspired by the pheromone-based communication strategy of ants, we introduce the concept of digital pheromones and design a digital pheromone based patrolling algorithm for robots. In our scheme, different events have different priorities. We use digital pheromones to model events priorities, which are corresponding to different values of pheromone and the values becomes weakening according to time. Robots are correspondingly modeled as ants that try to follow the route with largest pheromone strength. That is, robots choose the path with higher priority events. Once an event is detected, a message related to it will be sent to the nearest sink. In the path to sink, all relay nodes keep a record of the message and a trail of pheromone are formed which indicate the priority of the event which the pheromone will becomes weaker with time. Meanwhile, in each sensor, several different event messages may be recorded because several messages may be relayed by the same node. The total digital pheromone value on a 
segment of road is the accumulated value of all relayed messages. The main difference between digital pheromone and real pheromone is that digital pheromone is generated when an event is detected and relayed. When robots try to figure out their patrolling path, they will first communicate with sensors and sinks to find the route with the largest pheromone value. Usually, robots need to make a choice of next routine at an intersection of several roads. In our study, our concern is robots' patrolling strategy in a large region surveillance system but not the way how they handle events. The rest of this paper is organized as follows: In Section II, we introduce the concept of digital pheromones as inspired by the pheromone deployment of ants and the scent marking activity of animals. In Section III, we propose the digital pheromone based patrolling algorithm of robots. In Section IV, we provide simulation results. Finally in Sections V, we conclude the paper.

\section{Digital Pheromone}

Animals, like ants and primates depend on pheromones or scent marking to communicate. Scent marking is an activity among primates, and carries information to show their aggressiveness, or to establish and maintain their social rank. Pheromones and scent marking are both means of communication and collaboration. Robots also need to communicate and collaborate with their partners. Inspired by the pheromone based communication of ants, we introduce digital pheromone to denote the priorities of events. In our design, digital pheromone is used to describe the priority or importance of events. As an important feature of pheromone or scent marking, their strength weakens over time. Respectively, the priority of each event also degrades over time, and this is modeled as the decrease of digital pheromone. Digital pheromone is set up once an event is detected and decrease following a corresponding function to refer to real time priority. Meanwhile, each message has a lifetime, which died when its priority value degrades to zero. Although many sensors are connected, some are isolated. For a connected sensor/node, it can send messages and related digital pheromones to the nearest sink node. All relay nodes keep a record of the digital pheromone, which is calculated in phase with the original sensor. Meanwhile, in each sensor, all relayed messages as well as their digital pheromones are recorded. When an isolated sensor/node detects an event it keeps a record of the event until robots collect it or the priority value of the event degrades to zero. When a robot patrols to an intersection, it first communicates with sensors or sinks nearby and collects digital pheromones on each path. Assume that there are $n$ paths at an intersection. A robot will choose the path with the largest digital pheromone value. If the value is not available on some roads, robots will make decision based on available values. If none of the values are available, robots will randomly choose an unpatrolled one. Robots are patrolling to handle events based on digital pheromone collected from sensors. Once a message is collected by a robot, it is unnecessary to be kept any longer in sensors. Therefore, we assume that a sensor will clear its record of a digital pheromone if it is collected by a robot. All collected events are recorded in robots' memory. Once an event is handled, robots will report to sinks to update related information.

\section{Digital Pheromone Based Patrolling Algorithm}

In this section, we design two patrolling algorithms based on digital pheromone. The first design is Greedy Patrol Algorithm. Robots patrol randomly to handle events without specific destinations or responsible area. They choose the most efficient path according to the strength of digital pheromones. The second design is that robots are assigned a specific responsible area. Robots will first go to their responsible area and then start patrolling and handling events within the area.

\section{A. Greedy Search Algorithm:}

In this subsection, we propose the digital pheromone-based greedy search algorithm. Two different designs are provided: a local greedy search algorithm and a global greedy search algorithm. The first design is a local greedy search algorithm. Assume that there are 1,2 ..n paths available and their corresponding digital pheromones are $\mathrm{P} 1, \mathrm{P} 2 \ldots$ Pn. The next patrolling path is thus path $\square \square$, where $\mathrm{Pk} \square \square \max (\mathrm{P} 1, \mathrm{P} 2, \square, \mathrm{Pn})$. For example in Fig. 1, "AB" will be chosen if "AC" has lower digital pheromone value assuming that both "AB" and "AC" are unpatrolled road. The other design is a global greedy search algorithm, which is designed with a long-term consideration of distant digital pheromone values. That is, robots not only evaluate the digital pheromone of the next segment of road, but also next two or three segments. The main consideration of this design is that, robots may miss important events two or three segments away. In this design, the descending of pheromones should also be considered. Some events have high priority but far away. Their priorities may have decreased a lot when robots can finally reach them. Therefore, robots have to evaluate real time pheromone value when they finally arrive at events. We use $f(t)=a-b * t$ to model the decreasing of digital pheromones. For a route including $i$ segments of roads (from road \#1, road \#2, road \#i), we assume that the time a robot spent on each road is $t 1, t 2, \ldots$ ti. Then, the estimated pheromone is $E=\Sigma j=1 . . i \mathrm{fj}(\Sigma \mathrm{k}=1 . . \mathrm{jtk})$. Several factors are necessary to calculate the estimated pheromone, such as robots' speeds, the topology, lifetime of events, etc. As an example shown in Fig. 1, we use a tree structure to model the topology of roads at an intersection A. Three 
further segments are evaluated. Fig. 1 shows all available roads and the number on each edge is the length of the road. If we assume a robot's speed is $v=1$, then the number is also the time required to patrol the road. For simplicity in this example, we assume the decreasing model on each segment is $f(t)=50-t$. Then, the estimated digital pheromone of each route is $E f A D=69.5, E f A G=63.8$, and $E f A G=43.8$. Therefore, the best route is $A-B-$ $D$.

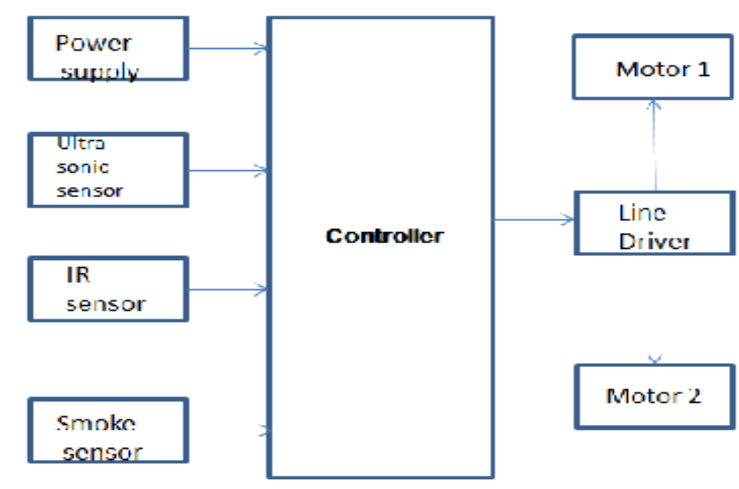

Fig.1a: Block Diagram of the patrolling robot

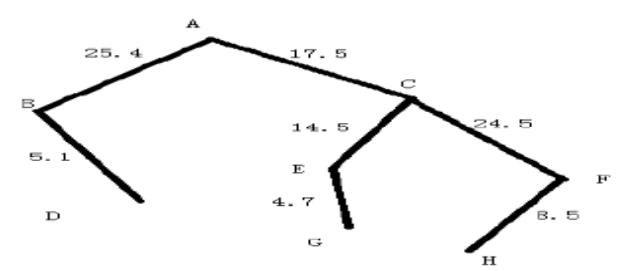

Fig. 1b. Roads at Intersection $A$

Robots calculate the best route every time when it reaches an intersection. At each intersection, the robot considers the total pheromone strength of next several segments, which is based on the specific algorithm design. Each calculation determines only one segment patrolling, which means that in Fig. 1, the robot will calculate the next step again when it arrives at B from A.

\section{B. Collaboration patrolling Algorithm}

Robots are organized to collaborate as a group when handling events within the network. The patrolling scheme plays a critical role in the collaboration among robots. There are two different considerations in our study. One consideration is that each robot is assigned a specific responsible area. Each robot is responsible for any events within its responsible area. In this situation, sensors report detected events to sinks. All events are then sent to a management center for scheduling further collaboration. The other consideration is that robots are not assigned specific areas but patrolling all around. In fact, the second consideration could be considered to be a special case of the first one. That is, each robot has the whole surveillance area as its responsible area. Initially, robots randomly patrol in their own area. They communicate through the assistance of network. A graph or topology is constructed based on the locations of events. Robots collaborate based on this graph. We assume that each robot is assembled with GPS that allows them to know their exact locations.

As they patrol, robots communicate with sensors to obtain updated event information and the topology. They also report handled events to sinks, and corresponding information is stored in sinks. We make the following assumptions:

1. When sensors detect events, they spread digital pheromones along the connection link. The digital pheromone related to the same event decreases as a function of time, $f(t)$. All relays of the message calculate its priority so that it is consistent with the original message. The total digital pheromone of a road is the accumulation of all events on the road.

2. Messages will be relayed to the nearest sink in order to avoid being flooded over the entire network. As at each intersection, there would be a sink deployed, each message will be stored by at most two sinks and only exists between these two sinks. However, nearby sinks can communicate with each other for digital pheromones on each section of roads.

3. Robots periodically communicate with sinks and the management center to upgrade the topology.

4. Once assigned a responsible area, a robot will target to the area through the shortest path based on topology. Furthermore, during this period, it will ignore all events on its way. 
5. Whenever a robot arrives at an intersection, it chooses one road at the intersection to patrol. It first communicates with the sink at the intersection to achieve pheromone values necessary for making a decision. The patrolling routes of robots are decided based on the greedy search algorithm.

6. After choosing a route to patrol, a robot will collect all messages on that road. If there are events on the road, the robot will handle all the events. After that all pheromones on that road are cleared.

\section{Responsibilities of Sensors}

Events and pheromone table records all live events and pheromones. The table includes all events detected or relayed by the sensors. The basic responsibility of sensors is to monitor surrounding environment. Whenever a sensor detects an event, it generates a digital pheromone corresponding to the event, which decreases with time following a function $f(t)$. It will then spread the event and its pheromone values along its connection link. After that, it maintains the pheromone table by decreasing the digital pheromone value over time. The second responsibility is to relay events and digital pheromones detected by other sensors. The transmission delay is considered to maintain of the pheromone table, and all pheromone values decrease over time. The third responsibility is to collaborate with robots. A sensor sends information about all events to robots passing by. Whenever digital pheromone is collected, the corresponding records in sensors will be cleared.

\section{A. Simulation Designs}

\section{Simulations}

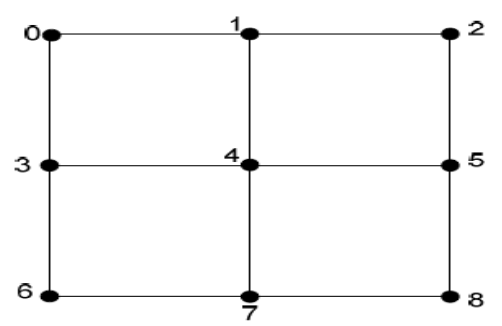

Fig. 2 A Simply Topology

In this subsection, we provide a simulation design to study our pheromone-based patrol algorithm. For simplicity, we use a simple topology as shown in Fig. 2 and make following assumptions.

1. Each vertex in the topology is a sink node. Sensors are deployed along edges. Robots also patrol along the edges.

2. Events are generated with uniform distribution of time and location. However, all events are generated on edges or vertices. Once an event occurs, a corresponding message will be generated and relayed to the nearest sink. We assume that, regardless of the exact location of an event on an edge, the corresponding message will be recorded by sensors on the whole edge from one vertex to the other.

3. For each event, all deployed RFIDs keep a consistent strength of the event. That is, for the same event, whenever an RFID is deployed, its original digital pheromone strength in the RFID is the same as the first deployed RFID tag. All the tags related to the same event have the same strength all the time.

4. When a robot arrives at a sink, it determines the strength of each edge from surrounding sensors. After comparison, it will choose the strongest edge with highest strength. After finishing its task on the edge, it will stop at the other end of the edge and determine the next path.

\section{B. Simulation results}

In this subsection, we demonstrate the results of our simulations. The local greedy patrolling algorithm is simulated and two different models of digital pheromones are considered. One is that digital pheromones remain constant since they are generated, and the other one is the decreasing model of digital pheromones. We use service rate (service time) to describe robots' work time on handling each event. In most of our simulations, the service time for handling each event is selected to be $f / 10, f / 20$, or $f / 50$, where $f$ denotes the digital pheromone value. Fig. 3 shows the percentage of handled events according to the speed of generating events. Three experiments are conducted with three service rates. These figures show the results when 100000 events occur. From the figure, we can learn that more events could be handled by a quick service and that more events could be handled if events do not frequently exist. However, when the service rate is very slow (e.g., f/10), the impact of the events' speed is hard to determine. Fig. 4 shows the performance of average delay of handled events compared to the speed of events. From the figure, we can learn that, when events are serviced quickly, average delay is small. Average delay also increases with the speed of events. But when service is slow $(f / 10)$, the impact of events' speed is hard to determine. 


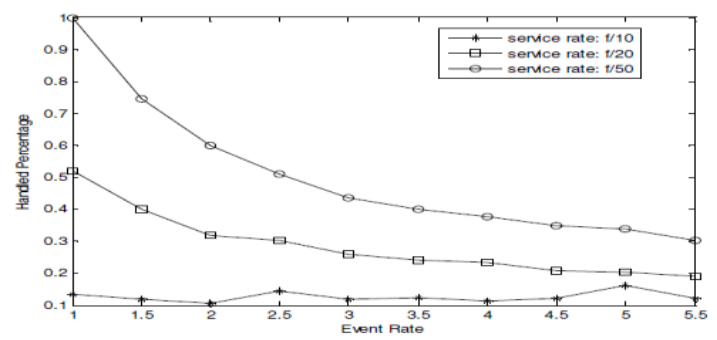

Fig. 3 Handled Percentage vs. Speed of Events (when event number=100000).

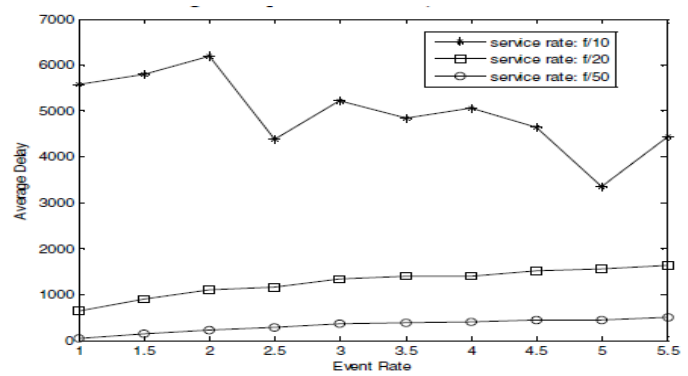

Fig. 4 Average Delay vs Speed of Events (when event number=100000).

Percentage vs. Speed of Events (when event number=100000).

\section{Conclusion}

Environment monitoring is an important application of wireless sensor networks. As a continuing work of our divide and conquer surveillance methodology we design this digital pheromone-based patrol scheme for robots. In our design, robots patrol paths with more high priority events in order to efficiently handle events detected in the monitored area. We also design simulations based on the patrolling strategy, through which we learn that the speed of generating events and the robot's handling time play important roles.

\section{References}

[1] Y. Xiao and Y. Zhang, "Divide- and conquer-based surveillance framework using robots, sensor nodes, and RFID tags," (Wiley) Wireless Communications and Mobile Computing (WCMC), accepted. DOI: 10.1002/wcm.863

[2] Y. Zhang, Y. Xiao, and K. L. Bales, "Primate Social Systems, Scent-marking, and Their Applications in Mobile and Static Sensor Networks," International Journal of Sensor Networks, Vol. 5, No. 4, 2009, pp. 210-222.

[3] Y. Zhang and Y. Xiao, "Primate-inspired Scent Marking for Mobile and Static Sensors and RFID Tags," Proceedings of 18th International Conference on Computer Communications and Networks, 2009 (ICCCN 2009).

[4] S.S., Chawathe, "Organizing Hot-Spot Police Patrol Routes", Intelligence and Security Informatics, 2007, pp. 79-86.

[5] C.T.Snowdon, C.H.Brown, and M.R.Peterson, "Primate Communication", Cambridge university press, pp. $283-293$.

[6] Y. Liang, J. Cao, L. Zhang, R. Wang, and Q. Pan, "A Biologically Inspired Sensor Wakeup Control Method for Wireless Sensor Networks", IEEE Transactions on Systems, Man, and Cybernetics, Part C: Applications and Reviews, vol. 4, issue: 5, pp. 525-538. Sept. 2010.

[7] S. Marwaha, J. Indulska, and M. Portmann, "Biologically Inspired Ant-Based Routing In Mobile Ad hoc Networks (MANET): A Survey", The Sixth International Conference on Ubiquitous Intelligence and Computing, July, 2009.

[8] M. G. Hinchey and R. Sterritt, "99\% (Biological) Inspiration...", Fourth IEEE International Workshop on Engineering of Autonomic and Autonomous Systems (EASe'07), March 2007.

[9] Z. Liu, M.Z. Kwiatkowska and C. Constantinou, "A biologically inspired congestion control routing algorithm for MANETs", Third IEEE International Conference on Pervasive Computing and Communications Workshops, 2005. PerCom 2005 Workshops, Mar. 2005, pp. 226-231.

[10] M. Dorigo and L.M. Gambardella, “Ant Colonies for the Traveling Salesman Problem,” BioSystems, 43:73-81, 1997.

[11] M. Dorigo and T. Stützle, Ant Colony Optimization, MIT Press, Cambridge, MA, 2004.

\section{About the authors}

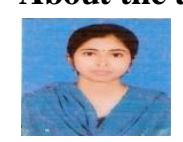

Lagusani .Sravanthi, M.Tech in DECS, Sri Kottam Tulasi Reddy

College Of Engineering \& Technology, S.K.T.R.M.C.E., A.P, India.

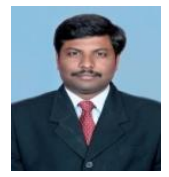

D.V.Srihari Babu, M.Tech (Ph.D.) Assoc.

Proff, Sri Kottam Tulasi Reddy College Of Engineering \& Technology, A.P, India. 